GANIT J. Bangladesh Math. Soc. (ISSN 1606-3694) 39 (2019) 101-110

DOI: https://doi.org/10.3329/ganit.v39i0.44167

\title{
INNER DERIVATIONS ON SEMIPRIME GAMMA RINGS
}

\author{
Sujoy Chakraborty ${ }^{1}$, Md. Mahbubur Rashid ${ }^{2}$ and Akhil Chandra Paul ${ }^{3}$ \\ ${ }^{1}$ Department of Mathematics, Shahjalal University of Science and Technology, Sylhet, Bangladesh \\ ${ }^{2}$ Department of Mathematics, Shahjalal University of Science and Technology, Sylhet, Bangladesh \\ ${ }^{3}$ Department of Mathematics, University of Rajshahi, Rajshahi, Bangladesh \\ E-mail: sujoy_chbty@yahoo.com ${ }^{1}$, mrashid_math@yahoo.com ${ }^{2}$, acpaulrubd_math@yahoo.com ${ }^{3}$
}

Received: 23-05-2019 Accepted: 11-11-2019

\begin{abstract}
We study inner derivations and generalized inner derivations in semiprime $\Gamma$-rings to develop some important results. If $f$ and $g$ are inner derivations of a semiprime $\Gamma$-ring $M$ satisfying the equation $f(x) \Gamma x+x \Gamma g(x)=0$ for all $x \in M$, then we show that $f=g=0$. This equation produces a number of results on generalized inner derivations as well.
\end{abstract}

Keywords: Generalized derivation, Inner derivation, Generalized inner derivation.

\section{Introduction}

For clear understanding and independency of this article we begin with the dentition of gamma ring along with some of its useful classifications and basic properties.

If $M$ and $\Gamma$ are additive abelian groups with a mapping $(a, \alpha, b) \rightarrow a \alpha b$ of $M \times \Gamma \times M \rightarrow M$ such that $(a+b) \alpha c=a \alpha c+b \alpha c, \quad a(\alpha+\beta) b=a \alpha b+a \beta b, \quad a \alpha(b+c)=a \alpha b+a \alpha c, \quad$ and $\quad(a \alpha b) \beta c$ $=a \alpha(b \beta c)$ for all $a, b, c \in M$ and $\alpha, \beta \in \Gamma$, then $M$ is called a $\Gamma$-ring. Throughout the article, $M$ represents a $\Gamma$-ring.

An element $x \in M$ is called a nilpotent element if, for all $\gamma \in \Gamma,(x \gamma)^{n} x=0$ for some positive integer $n . M$ is said to be 2-torsion free if $2 x=0$ implies $x=0$ for all $x \in M . M$ is called prime if $a \Gamma M \Gamma b=0$ (with $a, b \in M$ ) implies $a=0$ or $b=0$, whereas $M$ is called semiprime if $a \Gamma M \Gamma a=0$ (with $a \in M$ ) implies $a=0 . M$ is said to be commutative if $x \gamma y=y \gamma x$ holds for all $x, y \in M$ and $\gamma \in \Gamma$.

The set $Z(M)=\{c \in M: c \alpha m=m \alpha c$ for all $\alpha \in \Gamma$ and $m \in M\}$ is known as the center of $M$. If $a, b \in M$ and $\alpha \in \Gamma$, then $[a, b]_{\alpha}=a \alpha b-b \alpha a$ is called the commutator of $a$ and $b$ with respect to $\alpha$. It is clear that $M$ is commutative if and only if $[a, b]_{\alpha}=0$ for all $a, b \in M$ and $\alpha \in \Gamma$.

Some basic commutator identities are:

(i) $[a, b]_{\alpha}+[b, a]_{\alpha}=0$,

(c) GANIT: Journal of Bangladesh Mathematical Society, 2019

${ }^{1}$ Corresponding author 
(ii) $[a+b, c]_{\alpha}=[a, c]_{\alpha}+[b, c]_{\alpha}$,

(iii) $[a, b+c]_{\alpha}=[a, b]_{\alpha}+[a, c]_{\alpha}$,

(iv) $[a, b]_{\alpha+\beta}=[a, b]_{\alpha}+[a, b]_{\beta}$,

(v) $[a \beta b, c]_{\alpha}=a \beta[b, c]_{\alpha}+[a, c]_{\alpha} \beta b+a \beta(c \alpha b)-a \alpha(c \beta b)$, and

(vi) $[a, b \beta c]_{\alpha}=b \beta[a, c]_{\alpha}+[a, b]_{\alpha} \beta c+b \alpha(a \beta c)-b \beta(a \alpha c)$.

An additive map $d: M \rightarrow M$ is said to be a derivation of $M$ if $d(a \alpha b)=d(a) \alpha b+a \alpha d(b)$ holds for all $a, b \in M$ and $\alpha \in \Gamma$. It is called a reverse derivation of $M$ if $d(a \alpha b)=d(b) \alpha a+b \alpha d(a)$ holds for all $a, b \in M$ and $\alpha \in \Gamma$.

An additive map $f: M \rightarrow M$ is said to be a generalized derivation of $M$ if there exists a derivation $d: M \rightarrow M$ such that $f(a \alpha b)=f(a) \alpha b+a \alpha d(b)$ holds for all $a, b \in M$ and $\alpha \in \Gamma$.

A derivation $d$ is known as an innerderivation of $M$ if there exists $a \in M$ such that $d(x)=[a, x]_{\alpha}$ for all $x \in M$ and $\alpha \in \Gamma$. A generalized derivation $f$ is known as a generalized inner derivation of $M$ if there exist $a, b \in M$ such that $f(x)=a \alpha x+x \alpha b$ holds for all $x \in M$ and $\alpha \in \Gamma$.

A mapping $h: M \rightarrow M$ is called centralizing on $M$ if $[h(x), x]_{\alpha} \in Z(M)$ for all $x \in M$ and $\alpha \in \Gamma$ . In particular, if $[h(x), x]_{\alpha}=0$ for all $x \in M$ and $\alpha \in \Gamma$, then $h$ is called commuting on $M$.

M. Sapanci and A. Nakajima have introduced the notion of derivation of a $\Gamma$-ring in [11]. Afterwards, Y. Ceven and M. A. Ozturk [4] have introduced the notion of generalized derivation of a $\Gamma$-ring (we refer the reader to see [5] for detailed discussion and examples).

The study of centralizing and commuting maps on classical rings was initiated by E. C. Posner [10]. During the last three decades, a lot of research works has been performed to develop these concepts (e.g. [2, 3, 8]). We introduce those notions in case of gamma rings extensively.

This article aims to study some important properties of derivations and related maps on semiprime gamma rings. Considering a functional equation satisfied by inner derivations and generalized inner derivation on semiprime gamma rings, we establish some interesting results as applications of this equation. Especially, we obtain necessary and sufficient conditions for generalized inner derivations to be derivations and reverse derivations. We also obtain a commutativity theorem for a semiprime gamma ring additionally. We have been inspired to do this work from the results in [12] proved by A. B. Thaheem who has established the results in case of classical rings, whereas we extend those results for gamma rings with an additional condition.

\section{Main Results}

Throughout this section (almost everywhere), we may need to consider that a $\Gamma$-ring $M$ satisfies the condition

(*) $a \alpha b \beta c=a \beta b \alpha c$ for all $a, b, c \in M$ and $\alpha, \beta \in \Gamma$. 
If $M$ satisfies the condition (*), then the commutator identities (v) and (vi) respectively become

$$
\text { (V) }[a \beta b, c]_{\alpha}=a \beta[b, c]_{\alpha}+[a, c]_{\alpha} \beta b \text {, and (VI) }[a, b \beta c]_{\alpha}=b \beta[a, c]_{\alpha}+[a, b]_{\alpha} \beta c \text {. }
$$

Lemma 2.1 Let $M$ be a 2-torsion free semiprime $\Gamma$-ring satisfying the condition (*). If $a \in M$ be such that $a \alpha[a, x]_{\alpha}=[a, x]_{\alpha}$ aa for all $x \in M$ and $\alpha \in \Gamma$, then $a \in Z(M)$.

Proof. Define a mapping $d: M \rightarrow M$ by $d(x)=[a, x]_{\alpha}$ for all $x \in M$ and $\alpha \in \Gamma$. We claim that $d$ is a derivation on $M$; that is, $d(x \beta y)=d(x) \beta y+x \beta d(y)$ holds for all $x, y \in M$ and $\beta \in \Gamma$.

By the defining mapping, we have

$$
d(x \beta y)=[a, x \beta y]_{\alpha}=x \beta[a, y]_{\alpha}+[a, x]_{\alpha} \beta y=x \beta d(y)+d(x) \beta y,
$$

and therefore, $d$ is a derivation on $M$.

Now $d^{2}(x)=d(d(x))=[a, d(x)]_{\alpha}=\left[a,[a, x]_{\alpha}\right]_{\alpha}=a \alpha[a, x]_{\alpha}-[a, x]_{\alpha} \alpha a=0$ for all $x \in M$ (by hypothesis).

Since $d$ is a derivation on $M$, we have

$$
\begin{aligned}
0 & =d^{2}(x \beta y)=d(d(x \beta y))=d(d(x) \beta y+x \beta d(y)) \\
& =d^{2}(x) \beta y+d(x) \beta d(y)+d(x) \beta d(y)+x \beta d^{2}(y)=2 d(x) \beta d(y) .
\end{aligned}
$$

Since $M$ is 2-torsion free, $d(x) \beta d(y)=0$.

Putting $y=m \gamma x$ (for $m \in M$ and $\gamma \in \Gamma$ ), we obtain

$$
0=d(x) \beta d(m \gamma x)=d(x) \beta d(m) \gamma x+d(x) \beta m \gamma d(x)=d(x) \beta m \gamma d(x) .
$$

By the semiprimeness of $M$, we have $d(x)=0$.

That is, $[a, x]_{\alpha}=0$ for all $x \in M$ and $\alpha \in \Gamma$. This implies that $a \in Z(M)$.

Lemma 2.2 Let $M$ be a 2-torsion free semiprime $\Gamma$-ring satisfying the condition (*). If an additive mapping $f: M \rightarrow M$ is centralizing on $M$, then $f$ is commuting on $M$.

Proof.We have $[f(x), x]_{\alpha} \in Z(M)$ for all $x \in M$ and $\alpha \in \Gamma$. By linearizing this, we obtain

$$
[f(x), y]_{\alpha}+[f(y), x]_{\alpha} \in Z(M) \text { for all } x, y \in M \text { and } \alpha \in \Gamma .
$$

Replace $y$ by $x \beta x$ (for $x \in M$ and $\beta \in \Gamma$ ) to get $[f(x), x \beta x]_{\alpha}+[f(x \beta x), x]_{\alpha} \in Z(M)$.

Here, $[f(x), x \beta x]_{\alpha}=x \beta[f(x), x]_{\alpha}+[f(x), x]_{\alpha} \beta x$.

Since $[f(x), x]_{\alpha} \in Z(M)$, we find that $[f(x), x \beta x]_{\alpha}=2[f(x), x]_{\alpha} \beta x$. Thus,

(1) $2[f(x), x]_{\alpha} \beta x+[f(x \beta x), x]_{\alpha} \in Z(M)$.

By assumption, $[f(x \beta x), x \beta x]_{\alpha} \in Z(M)$ for all $x \in M$ and $\alpha, \beta \in \Gamma$. That is,

(2) $x \beta[f(x \beta x), x]_{\alpha}+[f(x \beta x), x]_{\alpha} \beta x \in Z(M)$. 
Taking fixed $x \in M$, let $z=[f(x), x]_{\alpha} \in Z(M)$ and $u=[f(x \beta x), x]_{\alpha}$.

We must show that $z=0$. By (1), we have

$$
\begin{aligned}
0 & =[f(x), 2 z \beta x+u]_{\alpha}=[f(x), 2 z \beta x]_{\alpha}+[f(x), u]_{\alpha} \\
& =2 z \beta[f(x), x]_{\alpha}+2[f(x), z]_{\alpha} \beta x+[f(x), u]_{\alpha}=2 z \beta z+[f(x), u]_{\alpha},
\end{aligned}
$$

where $2\left[f(x),[f(x), x]_{\alpha}\right]_{\alpha} \beta x=0$ since $[f(x), x]_{\alpha} \in Z(M)$.

Therefore, we get

(3) $[f(x), u]_{\alpha}=-2 z \beta z$.

According to (2), we have

$$
\begin{aligned}
0 & =[f(x), x \beta u+u \beta x]_{\alpha}=[f(x), x \beta u]_{\alpha}+[f(x), u \beta x]_{\alpha} \\
& =x \beta[f(x), u]_{\alpha}+[f(x), x]_{\alpha} \beta u+u \beta[f(x), x]_{\alpha}+[f(x), u]_{\alpha} \beta x .
\end{aligned}
$$

By (3), the above expression becomes $-2 x \beta z \beta z+z \beta u+u \beta z-2 z \beta z \beta x=0$,

which yields $2 z \beta u-4 z \beta z \beta x=0$, and therefore, $z \beta u=2 z \beta z \beta x$. From (3), we obtain (by using the last relation)

$$
\begin{aligned}
-2 z \beta z \beta z & =z \beta[f(x), u]_{\alpha}=[f(x), z \beta u]_{\alpha}-[f(x), z]_{\alpha} \beta u \\
& =[f(x), 2 z \beta z \beta x]_{\alpha}-[f(x), z]_{\alpha} \beta u=2 z \beta z \beta[f(x), x]_{\alpha}=2 z \beta z \beta z .
\end{aligned}
$$

Therefore, $4 z \beta z \beta z=0$. Hence, we get $(z \beta z)^{2} \beta z=0$. Since the centre of a semiprime $\Gamma$-ring contains no nonzero nilpotent elements (as we know), we conclude that $z=0$. This completes the proof. $\square$

Lemma 2.3 Let $M$ be a semiprime $\Gamma$-ring. If $a \in M$ is such that $x \alpha a \beta y=0$ for all $x, y \in M$ and $\alpha, \beta \in \Gamma$, then $a=0$.

Proof. We have $x \alpha a \beta y=0$. Multiplying it by $a \beta y$ on the left, we find that $(a \beta y) \gamma x \alpha(a \beta y)=0$ for all $\gamma \in \Gamma$. If we fix $y$, then the semiprimeness of $M$ forces that $a \beta y=0$. Since $y$ is arbitrary, we have $a \beta y \gamma a=0$ for all $y \in M$ and $\gamma \in \Gamma$. Again, by the semiprimeness of $M$, we obtain $a=0$. $\square$

Proposition 2.4 Let $M$ be a semiprime $\Gamma$-ring satisfying the condition (*), and let $a, b \in M$. Then the mapping $f: M \rightarrow M$ defined by $f(x)=a \alpha x+x \alpha b$ (with $x \in M$ and $\alpha \in \Gamma$ ) is a derivation if and only if $b=-a$.

Proof.For $x, y \in M$, we have $f(x+y)=a \alpha(x+y)+(x+y) \alpha b=a \alpha x+a \alpha y+x \alpha b+y \alpha b$

$$
=(a \alpha x+x \alpha b)+(a \alpha y+y \alpha b)=f(x)+f(y) .
$$

Therefore, $f$ is additive.

Now assume that $b=-a$, then $f(x)=a \alpha x-x \alpha a=[a, x]_{\alpha}$. Thus, we have 


$$
f(x \beta y)=[a, x \beta y]_{\alpha}=x \beta[a, y]_{\alpha}+[a, x]_{\alpha} \beta y=x \beta f(y)+f(x) \beta y,
$$

and therefore, $f$ is an inner derivation.

Conversely, suppose $f$ is a derivation. Then $f(x \beta y)=f(x) \beta y+x \beta f(y)$ for all $x, y \in M$ and $\beta \in \Gamma$.

Also, we have $f(x \beta y)=a \alpha x \beta y+x \beta y \alpha b$. Hence, by using the condition $(*)$, we get

$$
\begin{aligned}
a \alpha x \beta y+x \beta y \alpha b & =(a \alpha x+x \alpha b) \beta y+x \beta(a \alpha y+y \alpha b)=a \alpha x \beta y+x \alpha b \beta y+x \beta a \alpha y+x \beta y \alpha b \\
& =a \alpha x \beta y+x \alpha b \beta y+x \alpha a \beta y+x \beta y \alpha b=a \alpha x \beta y+x \alpha(b+a) \beta y+x \beta y \alpha b,
\end{aligned}
$$

and so, we obtain $x \alpha(b+a) \beta y=0$.

By Lemma 2.3, it follows that $b+a=0$, which implies $b=-a$.

In this case, $f(x)=a \alpha x-x \alpha a=[a, x]_{\alpha}$ for all $x \in M$ and $\alpha \in \Gamma$, and so, $f$ is then an inner derivation. $\square$

Remark 2.5 We make the following observations:

(i) If $M$ is a semiprime $\Gamma$-ring and $a \alpha x+x \alpha b=0$ for all $x \in M$ and $\alpha \in \Gamma$, then $a+b=0$; that is, $b=-a$.

This follows from Proposition 2.4, because the zero map is trivially a derivation.

(ii) If $a \Gamma$-ring $M$ is semiprime and $a \alpha x=x \alpha b$ for all $x \in M$ and $\alpha \in \Gamma$, then $a=b$.

Indeed, $a \alpha x=x \alpha b \Rightarrow a \alpha x-x \alpha b=a \alpha x+x \alpha(-b)=0$, and by (i), $a+(-b)=0$; that is, $a=b$.

(iii) If $M$ is a 2-torsion free semiprime $\Gamma$-ring such that a $\alpha x+x \alpha a=0$ for all $x \in M$ and $\alpha \in \Gamma$, then $a=0$.

Indeed, by (i), $a+a=0 \Rightarrow 2 a=0 \Rightarrow a=0$.

Proposition 2.6 Let $M$ be a 2-torsion free semiprime $\Gamma$-ring satisfying the condition $\left(^{*}\right)$, and let $a, b \in M$. Then the mapping $f: M \rightarrow M$ defined by $f(x)=a \alpha x+x \alpha b$ (for all $x \in M$ and $\alpha \in \Gamma)$ is a reverse derivation if and only if $f=0$.

Proof. It is clear that $f$ is additive. Assume that $f$ is a reverse derivation.

Then $f(x \beta y)=f(y) \beta x+y \beta f(x)$ for all $x, y \in M$ and $\beta \in \Gamma$.

So, we have

$$
a \alpha x \beta y+x \beta y \alpha b=(a \alpha y+y \alpha b) \beta x+y \beta(a \alpha x+x \alpha b)=a \alpha y \beta x+y \alpha b \beta x+y \beta a \alpha x+y \beta x \alpha b .
$$

Therefore,

$$
a \alpha(y \beta x-x \beta y)+(y \beta x-x \beta y) \alpha b+y \alpha b \beta x+y \beta a \alpha x=0 .
$$


So, we get the identity

(4) $a \alpha[y, x]_{\beta}+[y, x]_{\beta} \alpha b+y \alpha b \beta x+y \beta a \alpha x=0$.

Replacing $y$ by $x$ in (4), we obtain $x \alpha b \beta x+x \beta a \alpha x=0$ for all $x, y \in M$ and $\alpha, \beta \in \Gamma$.

By condition (*), it gives

(5) $x \alpha(b+a) \beta x=0$ for all $x \in M$ and $\alpha, \beta \in \Gamma$.

The linearization of (5) yields $(x+y) \alpha(b+a) \beta(x+y)=0$ for all $x, y \in M$ and $\alpha, \beta \in \Gamma$.

Therefore, $x \alpha(b+a) \beta x+x \alpha(b+a) \beta y+y \alpha(b+a) \beta x+y \alpha(b+a) \beta y=0$.

Using (5), we find

(6) $x \alpha(b+a) \beta y+y \alpha(b+a) \beta x=0$ for all $x, y \in M$ and $\alpha, \beta \in \Gamma$.

If we fix $x$ in (6), then by Remark 2.5(iii), we get $x \alpha(b+a+b+a) \beta x=0$.

Since $x$ is arbitrary, again by applying Remark 2.5(iii), we find that $2(b+a)=0$.

But, since $M$ is a 2-torsion free, we have $b=-a$.

Thus, $f(x)=a \alpha x-x \alpha a=[a, x]_{\alpha}$ for all $x \in M$ and $\alpha \in \Gamma$.

By assumption, $f$ is a reverse derivation, so

$$
f(x \beta y)=f(y) \beta x+y \beta f(x) \text { for all } x, y \in M \text { and } \beta \in \Gamma .
$$

That is, $[a, x \beta y]_{\alpha}=[a, y]_{\alpha} \beta x+y \beta[a, x]_{\alpha}$, and hence

$$
x \beta[a, y]_{\alpha}+[a, x]_{\alpha} \beta y=[a, y]_{\alpha} \beta x+y \beta[a, x]_{\alpha} .
$$

Replacing $x$ by $a$ in this last expression, we have

$$
a \beta[a, y]_{\alpha}=[a, y]_{\alpha} \beta a \text { for all } y \in M \text { and } \alpha, \beta \in \Gamma .
$$

In particular, $a \alpha[a, y]_{\alpha}=[a, y]_{\alpha} \alpha a$, and hence (by Lemma 2.1), we get $a \in Z(M)$.

Therefore, $f(x)=[a, x]_{\alpha}=0$ for all $x \in M$ and $\alpha \in \Gamma$, which shows that $f=0$.

The converse is obvious. $\square$

Theorem 2.7 Let $M$ be a semiprime $\Gamma$-ring satisfying the condition (*), and let $f, g: M \rightarrow M$ be inner derivations such that

(7) $f(x) \alpha x+x \alpha g(x)=0$ for all $x \in M$ and $\alpha \in \Gamma$.

Then $f=g=0$.

Proof. A linearization of (7) yields

$$
f(x+y) \alpha(x+y)+(x+y) \alpha g(x+y)=0 \text { for all } x, y \in M \text { and } \alpha \in \Gamma .
$$


Therefore, we get

$$
\begin{aligned}
0 & =(f(x)+f(y)) \alpha(x+y)+(x+y) \alpha(g(x)+g(y)) \\
& =f(x) \alpha x+f(x) \alpha y+f(y) \alpha x+f(y) \alpha y+x \alpha g(x)+x \alpha g(y)+y \alpha g(x)+y \alpha g(y) \\
& =(f(x) \alpha x+x \alpha g(x))+f(x) \alpha y+f(y) \alpha x+(f(y) \alpha y+y \alpha g(y))+x \alpha g(y)+y \alpha g(x) .
\end{aligned}
$$

By using (7) in the above expression, we obtain

(8) $f(x) \alpha y+f(y) \alpha x+x \alpha g(y)+y \alpha g(x)=0$.

Replacing $x$ by $y \beta x$ in (8), and using (*) and (7), we get

$$
\begin{aligned}
0 & =f(y \beta x) \alpha y+f(y) \alpha y \beta x+y \beta x \alpha g(y)+y \alpha g(y \beta x) \\
& =(f(y) \beta x+y \beta f(x)) \alpha y+f(y) \alpha y \beta x+y \beta x \alpha g(y)+y \alpha(g(y) \beta x+y \beta g(x)) \\
& =f(y) \beta x \alpha y+y \beta f(x) \alpha y+f(y) \alpha y \beta x+y \beta x \alpha g(y)+y \alpha g(y) \beta x+y \alpha y \beta g(x) \\
& =f(y) \beta x \alpha y+y \beta f(x) \alpha y+f(y) \alpha y \beta x+y \beta x \alpha g(y)+y \alpha g(y) \beta x+y \beta y \alpha g(x) \\
& =(f(y) \beta x \alpha y+y \beta x \alpha g(y))+y \beta(f(x) \alpha y+y \alpha g(x))+(f(y) \alpha y+y \alpha g(y)) \beta x \\
& =f(y) \alpha x \beta y+y \beta x \alpha g(y)+y \beta(f(x) \alpha y+y \alpha g(x)) .
\end{aligned}
$$

That is,

(9) $f(y) \alpha x \beta y+y \beta x \alpha g(y)+y \beta(f(x) \alpha y+y \alpha g(x))=0$.

By (8) and (9), we have

$$
\begin{aligned}
0 & =f(y) \alpha x \beta y+y \beta x \alpha g(y)-y \beta(f(y) \alpha x+x \alpha g(y)) \\
& =f(y) \alpha x \beta y+y \beta x \alpha g(y)-y \beta f(y) \alpha x-y \beta x \alpha g(y) \\
& =f(y) \alpha x \beta y-y \beta f(y) \alpha x .
\end{aligned}
$$

So, $[f(y) \alpha x, y]_{\beta}=0$ for all $x, y \in M$ and $\alpha, \beta \in \Gamma$. That is,

(10) $f(y) \alpha[x, y]_{\beta}+[f(y), y]_{\beta} \alpha x=0$.

Replacing $x$ by $x \gamma z$ in (10), we obtain

$$
\begin{aligned}
0 & =f(y) \alpha[x \gamma z, y]_{\beta}+[f(y), y]_{\beta} \alpha x \gamma z \\
& =f(y) \alpha x \gamma[z, y]_{\beta}+f(y) \alpha[x, y]_{\beta} \gamma z+[f(y), y]_{\beta} \alpha x \gamma z \\
& =f(y) \alpha x \gamma[z, y]_{\beta}+\left(f(y) \alpha[x, y]_{\beta}+[f(y), y]_{\beta} \alpha x\right) \gamma z .
\end{aligned}
$$

By using (10), we thus have

(11) $f(y) \alpha x \gamma[z, y]_{\beta}=0$.

Since $f$ is an inner derivation, we may assume that $f(y)=[a, y]_{\beta}$ for some $\alpha \in \Gamma$ and for all $\beta \in \Gamma$. 
Then by (11), we have $[a, y]_{\beta} \alpha x \gamma[z, y]_{\beta}=0$ for all $x, y, z \in M$ and $\alpha, \beta, \gamma \in \Gamma$.

In particular, if $z=a$, then we find that $[a, y]_{\beta} \alpha x \gamma[a, y]_{\beta}=0$ for all $x, y \in M$ and $\alpha, \beta, \gamma \in \Gamma$.

Since $M$ is semiprime, $[a, y]_{\beta}=0$ for all $y \in M$ and $\beta \in \Gamma$. This shows that $f=0$.

We now show that $g=0$. Since $f=0$, therefore, by (7), we have

(12) $x \alpha g(x)=0$ for all $x \in M$ and $\alpha \in \Gamma$.

Substituting $x$ by $x+y$ in (12), and using (12), we obtain

(13) $x \alpha g(y)+y \alpha g(x)=0$.

Replacing $x$ by $x \beta y$ in (13), and using (12), (13) and (*), we find that

Thus, we have

$$
\begin{aligned}
0 & =x \beta y \alpha g(y)+y \alpha g(x \beta y)=y \alpha g(x) \beta y+y \alpha x \beta g(y) \\
& =-x \alpha g(y) \beta y+y \alpha x \beta g(y)=-x \beta g(y) \alpha y+y \alpha x \beta g(y) \\
& =[y, x \beta g(y)]_{\alpha}=x \beta[y, g(y)]_{\alpha}+[y, x]_{\alpha} \beta g(y) .
\end{aligned}
$$

(14) $x \beta[y, g(y)]_{\alpha}+[y, x]_{\alpha} \beta g(y)=0$ for all $x, y \in M$ and $\alpha, \beta \in \Gamma$.

Now putting $z \gamma x$ for $x$ in (14), we obtain

$$
\begin{aligned}
0 & =z \chi x \beta[y, g(y)]_{\alpha}+[y, z \gamma x]_{\alpha} \beta g(y) \\
& =z \chi x \beta[y, g(y)]_{\alpha}+z \gamma[y, x]_{\alpha} \beta g(y)+[y, z]_{\alpha} \gamma x \beta g(y) \\
& =z \gamma\left(x \beta[y, g(y)]_{\alpha}+[y, x]_{\alpha} \beta g(y)\right)+[y, z]_{\alpha} \gamma x \beta g(y) .
\end{aligned}
$$

Using (14), we get

(15) $[y, z]_{\alpha} \gamma x \beta g(y)=0$ for all $x, y, z \in M$ and $\alpha, \beta, \gamma \in \Gamma$.

Since $g$ is an inner derivation, we may assume that $g(y)=[a, y]_{\alpha}$ for some $a \in M$ and for all $\alpha \in \Gamma$.

From (15), we have $[z, y]_{\alpha} \gamma x \beta[a, y]_{\alpha}=0$ for all $x, y, z \in M$ and $\alpha, \beta, \gamma \in \Gamma$.

In particular, if $z=a$, then we obtain $[a, y]_{\alpha} \gamma x \beta[a, y]_{\alpha}=0$, and by the semiprimeness of $M$, we conclude that $[a, y]_{\alpha}=0$ for all $\alpha \in \Gamma$. Since $y$ is arbitrary, we get $g(y)=[a, y]_{\alpha}=0$. It gives $g=0$.

Theorem 2.8 Let $M$ be a semiprime $\Gamma$-ring satisfying the condition (*), and let $h: M \rightarrow M$ be a generalized inner derivation defined by $h(x)=a \alpha x+x \alpha b \quad(x \in M, \alpha \in \Gamma)$ for some $a, b \in M$. Then $h$ is commuting if and only if $a, b \in Z(M)$.

Proof. Assume that $a, b \in Z(M)$.

We have $[h(x), x]_{\beta}=[a \alpha x+x \alpha b, x]_{\beta}=[a \alpha x, x]_{\beta}+[x \alpha b, x]_{\beta}$

$$
=a \alpha[x, x]_{\beta}+[a, x]_{\beta} \alpha x+x \alpha[b, x]_{\beta}+[x, x]_{\beta} \alpha b=[a, x]_{\beta} \alpha x+x \alpha[b, x]_{\beta}=0+0=0,
$$


since $a, b \in Z(M)$. Hence $h$ is commuting.

On the other hand, assume that $h$ is commuting. Then

$$
0=[h(x), x]_{\beta}=[a, x]_{\beta} \alpha x+x \alpha[b, x]_{\beta} \text { for all } x \in M \text { and } \beta \in \Gamma .
$$

Consider $f(x)=[a, x]_{\beta}$ and $g(x)=[b, x]_{\beta}$.

Then $f(x)$ and $g(x)$ are inner derivations of $M$ and $f(x) \alpha x+x \alpha g(x)=0$ for all $x \in M$ and $\alpha \in \Gamma$.

Therefore, by Theorem 2.7, $f=g=0$. That is, $[a, x]_{\beta}=0=[b, x]_{\beta}$ for all $x \in M$ and $\beta \in \Gamma$.

This implies that $a \in Z(M)$ and $b \in Z(M)$.

Theorem 2.9 Let $M$ be a 2-torsion free semiprime $\Gamma$-ring satisfying the condition (*), and let $h: M \rightarrow M$ be a generalized inner derivation defined by $h(x)=a \alpha x+x \alpha b \quad(x \in M, \alpha \in \Gamma)$ for some $a, b \in M$. Then $h$ is centralizing if and only if $a, b \in Z(M)$.

Proof. The mapping $h$ is clearly additive. Since $h$ is centralizing, by Lemma 2, we have $h$ is commuting on $M$. Therefore, by Theorem 2.8, $a, b \in Z(M)$. Conversely, we assume that $a, b \in Z(M)$. Then by Theorem $2.8, h$ is commuting, and hence it is centralizing. $\square$

Definition. $A \Gamma$-ring $M$ is said to be anticommutative if $\langle x, y\rangle_{\alpha}=x \alpha y+y \alpha x=0$ for all $x, y \in M$ and $\alpha \in \Gamma$.

Here we show that if $M$ is an anticommutative semiprime $\Gamma$-ring, then $M$ is commutative, i.e. $[x, y]_{\alpha}=0$ for all $x, y \in M$ and $\alpha \in \Gamma$.

Theorem 2.10 Let $M$ be a semiprime $\Gamma$-ring satisfying the condition (*). If $M$ is anticommutative, then $M$ is commutative.

Proof. We have $\langle x, y\rangle_{\alpha}=0$ for all $x, y \in M$ and $\alpha \in \Gamma$. Fix $y$ and define a mapping $h: M \rightarrow M$ by $h(x)=<x, y>_{\alpha}$. Then $h(x)=0$ for all $x \in M$. Therefore, $[h(x), x]_{\beta}=0$. This shows that $h$ is commuting. So, by Theorem 2.8, $y \in Z(M)$. Since $y$ is arbitrary, we conclude that $M$ is commutative.

\section{Acknowledgement}

The authors gratefully acknowledge the active support of the Research Grant 2015-2016 provided by Shahjalal University of Science and Technology Research Centre during this research work.

\section{REFERENCES}

[1] W. E. Barnes, On the T-rings of Nobusawa, Pacific J. Math., 18 (1966), 411-422.

[2] H. E. Bell and W. S. Martindale, Centralizing mappings of semiprime rings, Canad. Math. Bull., 30 (1987), 92-102.

[3] M. Bresar, Centralizing mappings and derivations in semiprime rings, J. Algebra, 156 (1993), 385-394. 
[4] Y. Ceven and M. A. Ozturk, On Jordan generalized derivations in gamma rings, Hacettepe J. Math. and Stat., 33 (2004), 11-14.

[5] S. Chakraborty and A. C. Paul, On Jordan generalized k-derivations of 2-torsion free prime $\Gamma_{N}$-rings, Internat. Math. Forum, 2(57) (2007), 2823-2829.

[6] N. Herstein, Topics in ring theory, University of Chicago Press, Chicago, 1969.

[7] B. Hvala, Generalized derivations in rings, Comm. Algebra, 26 (1998), 1147-1166.

[8] J. Mayne, Centralizing mappings of prime rings, Canad. Math. Bull., 27 (1984), 122-126.

[9] N. Nobusawa, On a generalization of the ring theory, Osaka J. Math., 1 (1964), 81-89.

[10] E. C. Posner, Derivations in prime rings, Proc. Amer. Math. Soc., 8 (1957), 1093-1100.

[11] M. Sapanci and A. Nakajima, Jordan derivations on completely prime gamma rings, Math. Japonica, 46(1) (1997), 47-51.

[12] A. B. Thaheem, On some properties of derivations on semiprime rings, Southeast Asian Bull. Math., 29 (2005), 1143-1152.

[13] N. Rehman, On generalized derivations as homomorphisms and anti-homomorphisms, Glasnik Mat., 39(59) (2004), 27-30. 